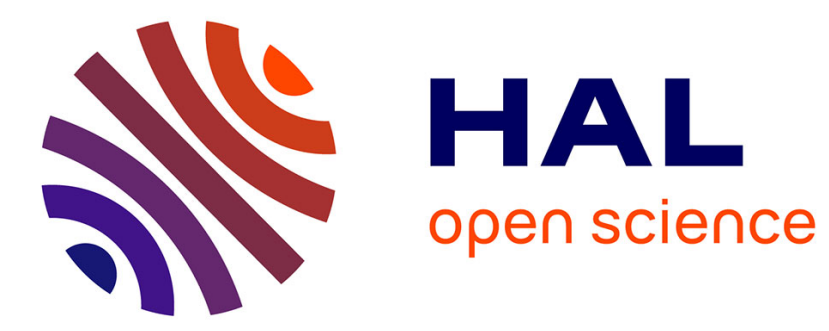

\title{
Closed Form Inverse of Local Multi-Trace Operators
}

\author{
Alan Ayala, Xavier Claeys, Victorita Dolean, Martin J Gander
}

\section{To cite this version:}

Alan Ayala, Xavier Claeys, Victorita Dolean, Martin J Gander. Closed Form Inverse of Local MultiTrace Operators. Domain Decomposition Methods in Science and Engineering XXIII, Jul 2015, Jeju island, South Korea. 10.1007/978-3-319-52389-7_9 . hal-01427610

\section{HAL Id: hal-01427610 https://hal.science/hal-01427610}

Submitted on 10 Jan 2017

HAL is a multi-disciplinary open access archive for the deposit and dissemination of scientific research documents, whether they are published or not. The documents may come from teaching and research institutions in France or abroad, or from public or private research centers.
L'archive ouverte pluridisciplinaire HAL, est destinée au dépôt et à la diffusion de documents scientifiques de niveau recherche, publiés ou non, émanant des établissements d'enseignement et de recherche français ou étrangers, des laboratoires publics ou privés. 


\title{
Closed Form Inverse of Local Multi-Trace Operators
}

\author{
Alan Ayala ${ }^{1}$, Xavier Claeys ${ }^{1}$, Victorita Dolean ${ }^{2}$, and Martin J. Gander ${ }^{3}$
}

\section{Introduction}

Local multi-trace operators arise when one uses a particular integral formulation for a transmission problem. A transmission problem for a second order elliptic operator is a problem defined on a domain which is decomposed into non-overlapping subdomains, but instead of imposing the continuity of the traces of the solution and their normal derivative along the interfaces between the subdomains, given jumps are imposed along the interfaces. The solution of a transmission problem is thus naturally discontinuous along the interfaces, and hence a domain decomposition formulation is imposed by the problem.

A local multi-trace formulation represents the solution in each subdomain using an integral formulation, and couples these solutions imposing the given jumps in the traces of the solution and the normal derivatives along the interfaces (hence the name multi-trace). This formulation was introduced in [9] to tackle transmission problems for the Helmholtz equation, where the material properties are constant in each subdomain, see also [4, 5], and [6] for associated boundary integral methods. Multi-trace formulations lead naturally to block preconditioners, see [10]. In [7], a simple introduction to local multitrace formulations is given in the language of domain decomposition, and it is shown that these block preconditioners are equivalent to the simultaneous application of a Dirichlet-Neumann and a Neumann-Dirichlet method to the transmission problem. Block preconditioners based on multi-trace formulations have also the potential to lead to nil-potent iterations, a more recent area of research in domain decomposition [1], and it was shown that for two subdomains, they correspond to optimal Schwarz methods, see [3].

Universié Pierre et Marie Curie, and INRIA Paris, France claeys@ann.jussieu.fr · University of Strathclyde, Glasgow, United Kingdom Victorita.Dolean@strath.ac.uk · University of Geneva, Switzerland martin.gander@unige.ch 


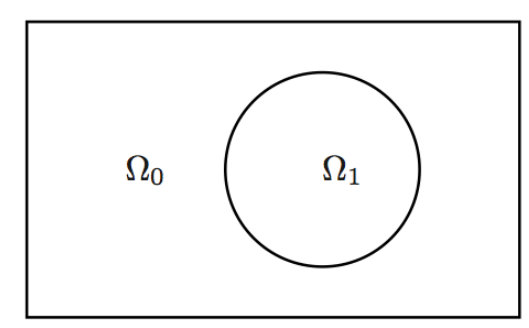

$\Omega_{2}$

Fig. 1 Geometrical configuration we consider in the analysis

We are interested here in the inverse of local multi-trace operators. We exhibit a closed form of this inverse for a model problem with three subdomains in the special case where the coefficients are homogeneous. An essential ingredient to obtain this closed form inverse are several remarkable identities which were recently discovered, see [3]. We illustrate our findings with a numerical experiment that shows that discretizing the closed form inverse gives indeed and approximate inverse of the discretized local multi-trace operator.

\section{Local Multi-Trace Formulation}

We start by introducing the local multi-trace formulation for a model problem. Consider a partition of the space $\mathbb{R}^{d}=\bar{\Omega}_{0} \cup \bar{\Omega}_{1} \cup \bar{\Omega}_{2}$ as shown in Figure 1. We assume that $\Omega_{j}, j=0,1,2$ are Lipschitz domains such that $\Omega_{j} \cap \Omega_{k}=\emptyset$ for $j \neq k$. Denoting by $\Gamma_{j}:=\partial \Omega_{j}$, we assume in addition that $\Gamma_{1} \cap \Gamma_{2}=\emptyset$ and $\Gamma_{0}=\Gamma_{1} \cup \Gamma_{2}$. Let $\boldsymbol{n}_{j}$ be the unit outer normal for $\Omega_{j}$ on its boundary $\Gamma_{j}$. For a sufficiently regular function $v$ we denote by $\left.v\right|_{\Gamma_{j}} ^{+}$the trace of $v$ and by $\left.\partial_{n_{j}} v\right|_{\Gamma_{j}} ^{+}$the trace of $\boldsymbol{n}_{j} \cdot \nabla v$ on $\Gamma_{j}$ taken from inside of $\Omega_{j}$. Similarly we define $\left.v\right|_{\Gamma_{j}} ^{-}$and $\left.\partial_{n_{j}} v\right|_{\Gamma_{j}} ^{-}$but with traces from outside of $\Omega_{j}$.

The elliptic transmission problem for which we want to study the local multi-trace formulation and its inverse is: find $u \in H^{1}\left(\mathbb{R}^{d}\right)$ such that

$$
\begin{aligned}
& -\Delta u+a_{j}^{2} u=0 \quad \text { in } \Omega_{j}, \quad j=0,1,2, \\
& {[u]_{\Gamma_{1}}=g_{1}, \quad[u]_{\Gamma_{2}}=g_{2},} \\
& {\left[\partial_{n} u\right]_{\Gamma_{1}}=h_{1}, \quad\left[\partial_{n} u\right]_{\Gamma_{2}}=h_{2}}
\end{aligned}
$$

where $a_{j}>0$ for $j=0,1,2, g_{j} \in H^{+1 / 2}\left(\Gamma_{j}\right)$ and $h_{j} \in H^{-1 / 2}\left(\Gamma_{j}\right)$ are given data of the transmission problem, and we used the classical jump notation for the Dirichlet and Neumann traces of the solution across the interfaces $\Gamma_{j}, j=1,2$, i.e. $[u]_{\Gamma_{j}}:=\left.u\right|_{\Gamma_{j}} ^{+}-\left.u\right|_{\Gamma_{j}} ^{-}$and $\left[\partial_{n} u\right]_{\Gamma_{j}}:=\left.\partial_{n_{j}} u\right|_{\Gamma_{j}} ^{+}-\left.\partial_{n_{j}} u\right|_{\Gamma_{j}} ^{-}$. 
Following [10], this problem can be rewritten as a boundary integral local multi-trace formulation, using the Calderón projector: let $\mathbb{H}\left(\Gamma_{j}\right):=$ $H^{1 / 2}\left(\Gamma_{j}\right) \times H^{-1 / 2}\left(\Gamma_{j}\right)$; then for $(g, h) \in \mathbb{H}\left(\Gamma_{j}\right)$, the Calderón projector $\mathbb{P}_{j}: \mathbb{H}\left(\Gamma_{j}\right) \rightarrow \mathbb{H}\left(\Gamma_{j}\right)$ interior to $\Omega_{j}$ associated to the operator $-\Delta+a_{j}^{2}$ is defined by

$$
\begin{aligned}
& \mathbb{P}_{j}(g, h):=\left(\left.v\right|_{\Gamma_{j}} ^{+},\left.\partial_{n_{j}} v\right|_{\Gamma_{j}} ^{+}\right) \text {where } v \text { satisfies } \\
& -\Delta v+a_{j}^{2} v=0 \text { in } \Omega_{j} \text { and in } \mathbb{R}^{d} \backslash \bar{\Omega}_{j}, \\
& {[v]_{\Gamma_{j}}=g \text { and }\left[\partial_{n} v\right]_{\Gamma_{j}}=h, \text { and }} \\
& \limsup _{|\boldsymbol{x}| \rightarrow \infty}|v(\boldsymbol{x})|<+\infty,
\end{aligned}
$$

and $\mathbb{P}_{j}$ is known to be a continuous map, see [12]. The decomposition $\Gamma_{0}=$ $\Gamma_{1} \cup \Gamma_{2}$ induces a natural decomposition of $\mathbb{P}_{0}$ in the following manner: for any $U \in \mathbb{H}\left(\Gamma_{0}\right)$ set $\rho_{j}(U):=\left.U\right|_{\Gamma_{j}} \in \mathbb{H}\left(\Gamma_{j}\right), j=1,2$. In addition, for any $V \in \mathbb{H}\left(\Gamma_{j}\right), j=1,2$, define $\rho_{j}^{*}(V) \in \mathbb{H}\left(\Gamma_{0}\right)$ by $\rho_{j}^{*}(V)=V$ on $\Gamma_{j}$ and $\rho_{j}^{*}(V)=0$ on $\Gamma_{0} \backslash \Gamma_{j}$. Then the projector $\mathbb{P}_{0}$ can be decomposed as

$$
\mathbb{P}_{0}=\left[\begin{array}{cc}
\tilde{\mathbb{P}}_{1} & \mathrm{R}_{1,2} / 2 \\
\mathrm{R}_{2,1} / 2 & \tilde{\mathbb{P}}_{2}
\end{array}\right], \quad \text { where }\left\{\begin{array}{l}
\tilde{\mathbb{P}}_{j}:=\rho_{j} \cdot \mathbb{P}_{0} \cdot \rho_{j}^{*}, \\
\mathrm{R}_{j, k} / 2:=\rho_{j} \cdot \mathbb{P}_{0} \cdot \rho_{k}^{*} .
\end{array}\right.
$$

The operators $\tilde{\mathbb{P}}_{j}: \mathbb{H}\left(\Gamma_{j}\right) \rightarrow \mathbb{H}\left(\Gamma_{j}\right)$ and $\mathrm{R}_{j, k}: \mathbb{H}\left(\Gamma_{k}\right) \rightarrow \mathbb{H}\left(\Gamma_{j}\right)$ are continuous. Following this decomposition, we identify $\mathbb{H}\left(\Gamma_{0}\right)$ with $\mathbb{H}\left(\Gamma_{1}\right) \times \mathbb{H}\left(\Gamma_{2}\right)$. We also introduce the sign switching operator $\mathrm{X}(v, q):=(v,-q)$, and a relaxation parameter $\sigma \in \mathbb{C} \backslash\{0\}$. The local multi-trace formulation of problem (1) is then: find $\left(U_{1}, U_{1}^{(0)}, U_{2}^{(0)}, U_{2}\right) \in \mathbb{H}\left(\Gamma_{1}\right)^{2} \times \mathbb{H}\left(\Gamma_{2}\right)^{2}$ such that

$$
\left[\begin{array}{cccc}
(1+\sigma) \mathrm{Id}-\mathbb{P}_{1} & -\sigma \mathrm{X} & 0 & 0 \\
-\sigma \mathrm{X} & (1+\sigma) \mathrm{Id}-\tilde{\mathbb{P}}_{1} & -\mathrm{R}_{1,2} / 2 & 0 \\
0 & -\mathrm{R}_{2,1} / 2 & (1+\sigma) \mathrm{Id}-\tilde{\mathbb{P}}_{2} & -\sigma \mathrm{X} \\
0 & 0 & -\sigma \mathrm{X} & (1+\sigma) \mathrm{Id}-\mathbb{P}_{2}
\end{array}\right] \cdot\left[\begin{array}{l}
U_{1} \\
U_{1}^{(0)} \\
U_{2}^{(0)} \\
U_{2}
\end{array}\right]=F
$$

where $F \in \mathbb{H}\left(\Gamma_{1}\right)^{2} \times \mathbb{H}\left(\Gamma_{2}\right)^{2}$ is some right-hand side depending on $g_{j}, h_{j}, \sigma$ whose precise expression is not important for our present study, where we want to obtain an explicit expression for the operator in (2) and its inverse for the special case

$$
a_{0}=a_{1}=a_{2} .
$$

To simplify the calculations when working with the entries of the operator in (2), we set $A_{j}:=-\mathrm{Id}+2 \mathbb{P}_{j}$ and $\tilde{A}_{j}:=-\mathrm{Id}+2 \tilde{\mathbb{P}}_{j}$. The following remarkable identities were established in $[3, \S 4.4]$ for the special case $(3): \mathbb{P}_{j}^{2}=\mathbb{P}_{j}, \tilde{\mathbb{P}}_{j}^{2}=$ $\tilde{\mathbb{P}}_{j}, \tilde{\mathbb{P}}_{1} \mathrm{R}_{1,2}=\tilde{\mathbb{P}}_{2} \mathrm{R}_{2,1}=0, \mathrm{XP}_{j} \mathrm{X}=\mathrm{Id}-\tilde{\mathbb{P}}_{j}$, and finally $\mathrm{R}_{1,2} \mathrm{R}_{2,1}=\mathrm{R}_{2,1} \mathrm{R}_{1,2}=$ 0 . These five properties can be reformulated in terms of the operators $A_{j}$, namely 
i) $A_{\tilde{\sim}}^{2}=\tilde{A}_{j}^{2}=\mathrm{Id}$,

ii) $\tilde{A}_{1} \mathrm{R}_{1,2}=-\mathrm{R}_{1,2}$ and $\tilde{A}_{2} \mathrm{R}_{2,1}=-\mathrm{R}_{2,1}$,

iii) $\mathrm{X} \cdot A_{j} \cdot \mathrm{X}=-\tilde{A}_{j}$,

iv) $\mathrm{R}_{1,2} \mathrm{R}_{2,1}=\mathrm{R}_{2,1} \mathrm{R}_{1,2}=0$,

v) $\mathrm{R}_{1,2} \tilde{A}_{2}=\mathrm{R}_{1,2}$ and $\mathrm{R}_{2,1} \tilde{A}_{1}=\mathrm{R}_{2,1}$.

Let us introduce auxiliary operators $\mathbb{A}, \Pi: \mathbb{H}\left(\Gamma_{1}\right)^{2} \times \mathbb{H}\left(\Gamma_{2}\right)^{2}$ defined by

$$
\mathbb{A}:=\left[\begin{array}{cccc}
A_{1} & 0 & 0 & 0 \\
0 & \tilde{A}_{1} & \mathrm{R}_{1,2} & 0 \\
0 & \mathrm{R}_{2,1} & \tilde{A}_{2} & 0 \\
0 & 0 & 0 & A_{2}
\end{array}\right], \quad \Pi:=\left[\begin{array}{cccc}
0 & \mathrm{X} & 0 & 0 \\
\mathrm{X} & 0 & 0 & 0 \\
0 & 0 & 0 & \mathrm{X} \\
0 & 0 & \mathrm{X} & 0
\end{array}\right] .
$$

According to property $i$ ) in $(4)$, we have $(\operatorname{Id}+\mathbb{A})^{2} / 4=(\operatorname{Id}+\mathbb{A}) / 2$, which implies the well known Calderón identity from the boundary integral equation literature, i.e.

$$
\mathbb{A}^{2}=\mathrm{Id},
$$

see for example [11, §4.4]. The local multi-trace operator on the left-hand side of Equation (2) can then be rewritten as

$$
\mathrm{MTF}_{\text {loc }}:=-\frac{1}{2} \mathbb{A}-\sigma \Pi+\left(\sigma+\frac{1}{2}\right) \mathrm{Id} .
$$

In (2), the terms associated with the relaxation parameter $\sigma$, namely Id $\Pi$, enforce the transmission conditions of problem (1). For $\sigma=0$, we have $\mathrm{MTF}_{\text {loc }}=\frac{1}{2}(\mathrm{Id}-\mathbb{A})$, which is a projector, and $\mathrm{MTF}_{\text {loc }}$ is thus not invertible. For $\sigma \neq 0$ however, $\mathrm{MTF}_{\text {loc }}$ was proved to be invertible in [2, Cor. 6.3]. The goal of the present contribution is to derive an explicit formula for the inverse of $\mathrm{MTF}_{\text {loc }}$, and we will thus assume $\sigma \neq 0$.

\section{Inverse of the Local Multi-Trace Operator}

We now derive a closed form inverse of the local multi-trace operator in (7) for the special case (3). Using that $\Pi^{2}=\mathrm{Id}$ and (6), we obtain

$$
\begin{aligned}
{[-\mathbb{A} / 2-\sigma \Pi} & +(\sigma+1 / 2) \operatorname{Id}][-\mathbb{A} / 2-\sigma \Pi-(\sigma+1 / 2) \operatorname{Id}] \\
& =(\mathbb{A} / 2+\sigma \Pi)^{2}-(\sigma+1 / 2)^{2} \mathrm{Id} \\
& =\left(\sigma^{2}+1 / 4-\sigma^{2}-\sigma-1 / 4\right) \operatorname{Id}+\sigma(\mathbb{A} \Pi+\Pi \mathbb{A}) / 2 \\
& =-\sigma \operatorname{Id}+\sigma(\mathbb{A} \Pi+\Pi \mathbb{A}) / 2 .
\end{aligned}
$$

Inspired by the calculations in $[3, \S 4.4]$ as well as [2, Prop. 6.1], we examine more closely $\mathbb{A} \Pi+\Pi \mathbb{A}$. We start by comparing $\mathbb{A} \Pi$ and $\Pi \mathbb{A}$ : 


$$
\mathbb{A} \Pi=\left[\begin{array}{cccc}
0 & A_{1} \mathrm{X} & 0 & 0 \\
\tilde{A}_{1} \mathrm{X} & 0 & 0 & \mathrm{R}_{1,2} \mathrm{X} \\
\mathrm{R}_{2,1} \mathrm{X} & 0 & 0 & \tilde{A}_{2} \mathrm{X} \\
0 & 0 & A_{2} \mathrm{X} & 0
\end{array}\right], \Pi \mathbb{A}=\left[\begin{array}{cccc}
0 & \mathrm{X} \tilde{A}_{1} & \mathrm{XR}_{1,2} & 0 \\
\mathrm{X} A_{1} & 0 & 0 & 0 \\
0 & 0 & 0 & \mathrm{X} A_{2} \\
0 & \mathrm{XR}_{2,1} & \mathrm{X} \tilde{A}_{2} & 0
\end{array}\right]
$$

According to Property iii) in (4), we have $\mathrm{X} \tilde{A}_{j}+A_{j} \mathrm{X}=0$ and $\mathrm{X} A_{j}+\tilde{A}_{j} \mathrm{X}=0$, and thus from (9) we obtain

$$
\Pi \mathbb{A}+\mathbb{A} \Pi=\left[\begin{array}{cccc}
0 & 0 & \mathrm{XR}_{1,2} & 0 \\
0 & 0 & 0 & \mathrm{R}_{1,2} \mathrm{X} \\
\mathrm{R}_{2,1} \mathrm{X} & 0 & 0 & 0 \\
0 & \mathrm{XR}_{2,1} & 0 & 0
\end{array}\right]
$$

Computing the square of this operator, and taking into account Property iv) from (4), we obtain

$$
(\Pi \mathbb{A}+\mathbb{A} \Pi)^{2}=\left[\begin{array}{cccc}
\mathrm{XR}_{1,2} \mathrm{R}_{2,1} \mathrm{X} & 0 & 0 & 0 \\
0 & \mathrm{R}_{1,2} \mathrm{R}_{2,1} & 0 & 0 \\
0 & 0 & \mathrm{R}_{2,1} \mathrm{R}_{1,2} & 0 \\
0 & 0 & 0 & \mathrm{XR}_{2,1} \mathrm{R}_{1,2} \mathrm{X}
\end{array}\right]=0 .
$$

From this we conclude that $(-\mathrm{Id}+(\mathbb{A} \Pi+\Pi \mathbb{A}) / 2)^{-1}=-\mathrm{Id}-(\mathbb{A} \Pi+\Pi \mathbb{A}) / 2$. Coming back to (8), we obtain a first expression for the inverse of the local multi-trace operator, namely

$$
\begin{aligned}
{[-\mathbb{A}} & / 2-\sigma \Pi+(\sigma+1 / 2) \operatorname{Id}]^{-1} \\
= & \sigma^{-1}[\mathbb{A} / 2+\sigma \Pi+(\sigma+1 / 2) \operatorname{Id}][\operatorname{Id}+(\mathbb{A} \Pi+\Pi \mathbb{A}) / 2] \\
= & \sigma^{-1}\left[\frac{1}{2}(1+\sigma) \mathbb{A}+(\sigma+1 / 4) \Pi+(\sigma+1 / 2)(\operatorname{Id}+(\mathbb{A} \Pi+\Pi \mathbb{A}) / 2)\right] \\
& +\sigma^{-1}\left[\frac{\sigma}{2} \Pi \mathbb{A} \Pi+\frac{1}{4} \mathbb{A} \Pi \mathbb{A}\right]
\end{aligned}
$$

The only terms that are not explicitly known yet in (10) are the last two, $\Pi \mathbb{A} \Pi$ and $\mathbb{A} \Pi \mathbb{A}$. Combining (9) with Definition (5), direct calculation yields

$$
\Pi \mathbb{A} \Pi=\left[\begin{array}{cccc}
-A_{1} & 0 & 0 & \mathrm{XR}_{1,2} \mathrm{X} \\
0 & -\tilde{A}_{1} & 0 & 0 \\
0 & 0 & -\tilde{A}_{2} & 0 \\
\mathrm{XR}_{2,1} \mathrm{X} & 0 & 0 & -A_{2}
\end{array}\right],
$$

and similarly, we also obtain

$$
\mathbb{A} \Pi \mathbb{A}=\left[\begin{array}{cccc}
0 & -\mathrm{X} & \mathrm{XR}_{1,2} & 0 \\
-\mathrm{X} & 0 & 0 & -\mathrm{R}_{1,2} \mathrm{X} \\
-\mathrm{R}_{2,1} \mathrm{X} & 0 & 0 & -\mathrm{X} \\
0 & \mathrm{XR}_{2,1} & -\mathrm{X} & 0
\end{array}\right]
$$

We have now derived an explicit expression for each term in (10), which leads to a close form matrix expression for the inverse of the local multi-trace 


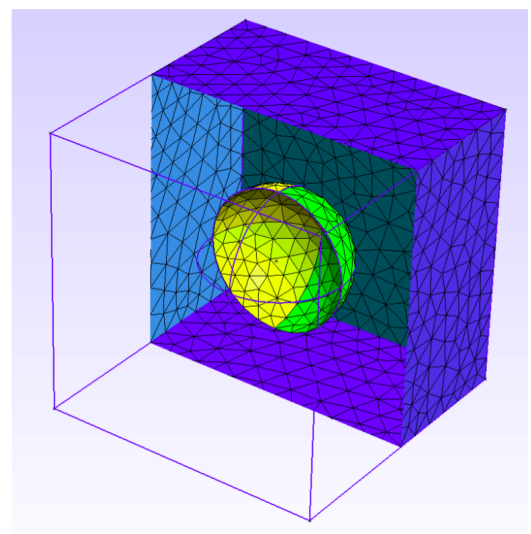

Fig. 2 3D geometry for the numerical experiment

operator, namely

$$
\mathrm{MTF}_{\text {loc }}^{-1}=\left(1+\frac{1}{2 \sigma}\right) \mathrm{Id}+\frac{1}{\sigma}\left[\begin{array}{llll}
\frac{1}{2} A_{1} & \sigma \mathrm{X} & \frac{\sigma+1}{2} \mathrm{XR}_{1,2} & \frac{\sigma}{2} \mathrm{XR}_{1,2} \mathrm{X} \\
\sigma \mathrm{X} & \frac{1}{2} \tilde{A}_{1} & \frac{\sigma+1}{2} \mathrm{R}_{1,2} & \frac{\sigma}{2} \mathrm{R}_{1,2} \mathrm{X} \\
\frac{\sigma}{2} \mathrm{R}_{2,1} \mathrm{X} & \frac{\sigma+1}{2} \mathrm{R}_{2,1} & \frac{1}{2} \tilde{A}_{2} & \sigma \mathrm{X} \\
\frac{\sigma}{2} \mathrm{XR}_{2,1} \mathrm{X} & \frac{\sigma+1}{2} \mathrm{XR}_{2,1} & \sigma \mathrm{X} & \frac{1}{2} A_{2}
\end{array}\right] .
$$

The expression $\mathrm{MTF}_{\mathrm{loc}} \cdot \mathrm{MTF}_{\mathrm{loc}}^{-1}=\mathrm{Id}$ should not be mistaken for the Calderón identity (6). The primary difference is that (11) involves coupling terms between $\Omega_{1}$ and $\Omega_{2}$, whereas in (6), all three subdomains are decoupled.

\section{Numerical Experiment}

We now illustrate the closed form inversion formula (11) for the local multitrace formulation by a numerical experiment. We consider a three dimensional version of the geometrical setting described at the beginning in Figure 1. Here $\Omega_{1}:=B(0,0.5)$ is the open ball centered at 0 with radius $0.5, \Omega_{2}:=$ $\mathbb{R}^{3} \backslash[-1,+1]^{3}$, and $\Omega_{0}:=\mathbb{R}^{3} \backslash \bar{\Omega}_{1} \cup \bar{\Omega}_{2}$, see Figure 2 .

For our numerical results, we discretize both $\mathrm{MTF}_{\text {loc }}$ given by (7) leading to a matrix we denote by $\left[\mathrm{MTF}_{\text {loc }}\right]$, and $\mathrm{MTF}_{\text {loc }}^{-1}$ given by (11) leading to a matrix denoted by $\left[\mathrm{MTF}_{\text {loc }}^{-1}\right]$. Our discretization using the code BEMTOOL ${ }^{1}$ is based on a Galerkin method where both Dirichlet and Neumann traces are approximated by means of continuous piece-wise linear functions on the same mesh. We use a triangulation with a mesh width $h=0.35$, and generated the mesh using GMsh, see [8].

\footnotetext{
${ }^{1}$ available on https://github.com/xclaeys/bemtool under Lesser Gnu Public License.
} 

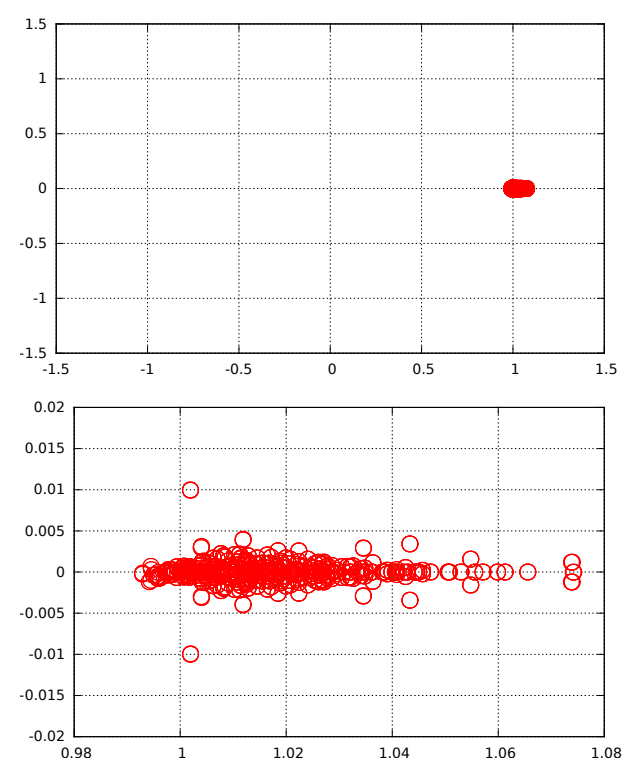

Fig. 3 Eigenvalues of the matrix $\mathrm{M}_{h}^{-1} \cdot\left[\mathrm{MTF}_{\text {loc }}\right] \cdot \mathrm{M}_{h}^{-1} \cdot\left[\mathrm{MTF}_{\text {loc }}^{-1}\right]$ for $\sigma=-\frac{1}{2}$, with a zoom below around 1 .

Let $\mathrm{M}_{h}$ be the mass matrix associated with the duality pairing used to write (2) in variational form. We represent the spectrum of the matrix $\mathrm{M}_{h}^{-1}$. $\left[\mathrm{MTF}_{\text {loc }}\right] \cdot \mathrm{M}_{h}^{-1} \cdot\left[\mathrm{MTF}_{\text {loc }}^{-1}\right]$ in Figure 3 . We see that the eigenvalues are clustered around 1, which agrees well with our analysis at the continuous level.

\section{Conclusions}

We have shown in this paper that it is possible for the local multi-trace operator of a model transmission problem to obtain a closed form for the inverse. This would therefore be an ideal preconditioner for local multi-trace formulations. We are currently investigating if such closed form inverses are also possible for more general situations, where the coefficients are only constant in each subdomain, and in the presence of more subdomains. The closed form inverse seems to be inherent to the formulation, and not dependent on the specific form of the partial differential equation.

Acknowledgement This work received support from the ANR research Grant ANR-15-CE23-0017-01. 


\section{References}

[1] F. Chaouqui, M. J. Gander, and K. Santugini-Repiquet. On nilpotent subdomain iterations. In C. Cai, D. Keyes, H. H. Kim, A. Klawonn, C.-O. Lee, E.-J. Park, and O. Widlund, editors, Domain Decomposition Methods in Science and Engineering XXIII, 2016.

[2] X. Claeys. Essential spectrum of local multi-trace boundary integral operators. IMA Journal of Applied Mathematics, 2016.

[3] X. Claeys, V. Dolean, and M. J. Gander. An Introduction to Multitrace Formulations and Associated Domain Decomposition Solvers. Preprint https://arxiv.org/abs/1605.04422, 2015.

[4] X. Claeys and R. Hiptmair. Electromagnetic scattering at composite objects: a novel multi-trace boundary integral formulation. ESAIM Math. Model. Numer. Anal., 46(6):1421-1445, 2012.

[5] X. Claeys and R. Hiptmair. Multi-trace boundary integral formulation for acoustic scattering by composite structures. Comm. Pure Appl. Math., 66(8):1163-1201, 2013.

[6] X. Claeys, R. Hiptmair, and E. Spindler. A second-kind Galerkin boundary element method for scattering at composite objects. BIT, 55(1):33$57,2015$.

[7] V. Dolean and M. J. Gander. Multitrace formulations and DirichletNeumann algorithms. In J. Erhel, M. J. Gander, L. Halpern, G. Pichot, T. Sassi, and O. Widlund, editors, Domain Decomposition Methods in Science and Engineering XXII. Springer, 2015.

[8] C. Geuzaine and J.-F. Remacle. Gmsh: A 3-D finite element mesh generator with built-in pre- and post-processing facilities. Internat. J. Numer. Methods Engrg., 79(11):1309-1331, 2009.

[9] R. Hiptmair and C. Jerez-Hanckes. Multiple traces boundary integral formulation for Helmholtz transmission problems. Adv. Comput. Math., 37(1):39-91, 2012.

[10] R. Hiptmair, C. Jerez-Hanckes, J.-F. Lee, and Z. Peng. Domain decomposition for boundary integral equations via local multi-trace formulations. In J. Erhel, M. J. Gander, L. Halpern, G. Pichot, T. Sassi, and O. Widlund, editors, Domain Decomposition Methods in Science and Engineering XXI, pages 43-57. Springer, 2014.

[11] J.-C. Nédélec. Acoustic and electromagnetic equations, volume 144 of Applied Mathematical Sciences. Springer-Verlag, New York, 2001. Integral representations for harmonic problems.

[12] S. A. Sauter and C. Schwab. Boundary element methods, volume 39 of Springer Series in Computational Mathematics. Springer-Verlag, Berlin, 2011. 